\title{
Hospitales amigables para adolescentes: preferencias de los pacientes ${ }^{1}$
}

\author{
Ana M Ullán ${ }^{1}$ \\ Isabel Serrano ${ }^{2}$ \\ Marta Badía ${ }^{2}$ \\ Juan Delgado ${ }^{3}$
}

${ }^{1}$ Departamento de Psicología Social y Antropología, Universidad de Salamanca

${ }^{2}$ Departamento de Personalidad, Evaluación y Tratamientos Psicológicos, Universidad de Salamanca

${ }^{3}$ Departamento de Psicología Básica, Psicobiología y Metodología de las Ciencias del Comportamiento, Universidad de Salamanca

Dirección postal y correo electrónico del autor para la correspondencia:

Ana M Ullán.

Facultad de Ciencias Sociales. Campus Miguel de Unamuno. 37007. Salamanca.

ullan@usal.es

Financiación:

Esta investigación fue financiada por la Secretaría de Estado de Universidades del Ministerio de Ciencia e Innovación (ref. EDU2008-05441-C02-02) y la Junta de Castilla y León (ref. SA077A07).

\footnotetext{
${ }^{1}$ Este artículo es una versión final del publicado en la revista Enfermería Clínica, cuya referencia completa es Ullan AM, Serrano I, Badía M, Delgado J. Hospitales amigables para adolescentes: preferencias de los pacientes. Enfermería Clínica. 2010;20(6):341. DOI: $10.1016 /$ j.enfcli.2010.07.006.
} 


\section{Hospitales amigables para adolescentes: preferencias de los pacientes}

\section{Resumen}

Objetivo: Conocer las preferencias y prioridades de adolescentes hospitalizados sobre potenciales líneas de mejora de su estancia en el hospital.

Método: Estudio prospectivo transversal descriptivo. Los participantes respondieron un cuestionario sobre preferencias y prioridades en la hospitalización que incluía datos de edad, sexo y experiencia de hospitalización, 15 preguntas (escala Likert) para valorar de 1 a 5 la importancia concedida a diferentes aspectos de la estancia hospitalaria, 6 preguntas con varias opciones de respuesta sobre las preferencias por tipo y compañero de habitación y por acompañante nocturno en el hospital, y 5 preguntas abiertas para indicar aspectos positivos y negativos de la hospitalización y sugerencias para mejorarla.

Resultados: Respondieron el cuestionario 96 adolescentes, $76 \%$ de los elegibles. La amabilidad del personal sanitario y que no se retrasaran las pruebas médicas fueron los aspectos cuya importancia para mejorar la hospitalización de los adolescentes fue más valorada por ellos. De tener que compartir la habitación del hospital, el $86.4 \%$ (IC95\%:77.9-92.5) preferían hacerlo con alguien de edad similar. Entre las sugerencias de mejora de la hospitalización efectuadas destacaron las relativas a los servicios de ocio para pacientes adolescentes en el hospital (56.2\% de las referencias). Como aspecto positivo de la hospitalización el mayor número de referencias $(50.0 \%)$ tuvo que ver con el trato del personal sanitario.

Conclusiones: Los adolescentes manifiestan una perspectiva de usuarios del hospital que debe tenerse en consideración para desarrollar líneas de mejora de los servicios de salud que reciben.

Palabras clave: Adolescentes hospitalizados; Calidad Servicios de Salud; Participación de los pacientes; Episodios de cuidado; Cuidados centrados en el paciente; Administración del Hospital. 


\title{
Friendly hospitals for adolescents: patients' preferences
}

\begin{abstract}
Objective: To determine the preferences and priorities of a sample of hospitalized adolescents about potential ways to improve their stay in the hospital.

Method: We performed a prospective, cross-sectional, descriptive study. The participants completed a questionnaire of their preferences and priorities regarding hospitalization, which included data about age, sex, and hospitalization experience, 15 questions (Likert-type scale) to rate from 1 to 5 the importance they grant to diverse aspects of the stay at hospital, 6 questions with various response options about their preferences concerning roommates and accompanying person at night in the hospital, and 5 open questions to indicate positive and negative aspects about hospitalization and suggestions to improve it.
\end{abstract}

Results: 96 adolescents completed the questionnaire, which was $76 \%$ of the eligible sample. The importance of agreeableness of clinical staff and for medical tests not to suffer any delay were the aspects that were considered most important to improve adolescents' hospitalization . If they had to share the hospital room, $86.4 \%$ (95\% CI: 77.9-92.5) preferred to share with someone of the same age. Among the noteworthy suggestions to improve hospitalization were those involving leisure services in the hospital for adolescent patients (56.2\% of the references). As a positive aspect of hospitalization, most of the references $(50.0 \%)$ referred to the relationship with the clinical staff.

Conclusions: The adolescents express a hospital user perspective that should be taken into account to develop improvements of the health care they receive.

Key words: Adolescent, Hospitalized; Quality of Health Services; Patient Participation; Episode of Care; Patient-Centered Care; Hospital Administration 


\section{Introducción}

El desarrollo de modelos de atención centrados en el paciente es un cambio importante producido en el ámbito del cuidado de la salud en los últimos años ${ }^{(1)}$. Estos modelos se definen como respetuosos y conscientes de las preferencias, de las necesidades y de los valores individuales de los pacientes y requieren un esfuerzo por mejorar la sensibilidad hacia estas necesidades y experiencias de las personas en relación con el cuidado de su salud ${ }^{(2,3)}$. Un punto clave para el desarrollo de estos modelos es la incorporación de la perspectiva de los pacientes en el diseño de los sistemas de cuidado. Entre las aproximaciones metodológicas utilizadas para incorporar esta perspectiva de los pacientes destaca la identificación de las preferencias de los usuarios en los episodios de cuidado de la salud ${ }^{(4)}$. En esta línea de atender las perspectivas de los pacientes se han estudiado las preferencias y prioridades de adolescentes, enfermos crónicos o no, en relación con cuestiones generales del cuidado de su salud y de la atención médica que reciben. Tambien se han estudiado las preferencias de los adolescentes por diversos aspectos del diseño de los hospitales y su satisfacción respecto a varias necesidades básicas cuando están hospitalizados.

Partiendo de la base de que el personal sanitario debe comprender las prioridades de los pacientes para crear un modelo de tratamiento efectivo, Britto et al. comprobaron que las visiones de los especialistas sobre el cuidado de la salud no

siempre coinciden con la perspectiva de los adolescentes a los que atienden ${ }^{(2)}$. Estos autores llevaron a cabo una investigación con adolescentes con enfermedades crónicas y con médicos especialistas de las clínicas donde eran atendidos los adolescentes. Los adolescentes valoraron la importancia que para ellos tenían a una serie de ítems relacionados con la calidad del cuidado y con el estilo de comunicación médicopaciente. Los médicos, por su parte, valoraron la importancia que, según ellos, los adolescentes daban a estos aspectos. Para los ítems de calidad del cuidado, las correlaciones entre las respuestas de los adolescentes y las de los médicos fueron altas y ambos grupos dieron la máxima prioridad a los ítems relacionados con el manejo del dolor. Sin embargo, los médicos subestimaron la importancia que los adolescentes concedían a la comunicación amistosa con los doctores y a los aspectos médico-técnicos 
del cuidado. Los autores concluyeron que los médicos comprendían la importancia que los adolescentes con enfermedades crónicas daban al manejo del dolor, pero sobreestimaban el nivel de autonomía deseado por los adolescentes. Según los autores, preguntar a los adolescentes por sus preferencias podía ser el primer paso para mejorar su experiencia de cuidado. A conclusiones similares llegan otros investigadores que han analizado la las actitudes y las preferencias de grupos de adolescentes en relación con el cuidado de la salud. Desde una aproximación exploratoria Wray y Maynard ${ }^{(5)}$ trataron de comprender mejor las necesidades y expectativas de jóvenes pacientes con enfermedades cardiacas congénitas con el fin de identificar buenas prácticas y áreas en las que se pudieran mejorar los procesos de comunicación y las instalaciones disponibles para estos pacientes, así como para aumentar la comprensión de los procesos de transición entre los servicios pediátricos y los servicios de adultos. Utilizaron un cuestionario postal enviado a jóvenes de entre 17 y 20 años con patologías cardiacas crónicas. En el cuestionario se incluyeron preguntas abiertas sobre la experiencia de los pacientes en servicios de cardiología pediátrica y en servicios de adultos, su percepción de la transición entre los servicios y sugerencias de mejora. Se efectuó un análisis cualitativo de las respuestas de los sujetos y los resultados indicaron que había temas, como la comunicación, la interacción en los procesos de cuidado y aspectos relativos al entorno que tenían que ver con la satisfacción o insatisfacción de los jóvenes con la atención que recibían en los servicios pediátricos o en los servicios de adultos. La conclusión de los autores fue que los jóvenes querían una aproximación individualizada a su cuidado que reconociera sus necesidades prácticas y emocionales específicas, y que era necesario tener en cuenta la perspectiva de los jóvenes durante todas las etapas por las que pasan como pacientes.

Trabajando también con adolescentes con enfermedades crónicas, Jedeloo et al. defendieron en su estudio una aproximación a las actitudes y preferencias de los adolescentes que no estuviera determinada por la enfermedad específica que padecían (6). Según ellos, los adolescentes con diferentes problemas médicos comparten muchos aspectos de sus experiencias subjetivas relacionadas con su condición crónica, con su tratamiento y su salud. Utilizaron en su estudio una metodología diseñada para identificar similitudes y diferencias en actitudes hacia el manejo de la enfermedad. Esta metodología permitió definir perfiles de adolescentes que, con una variedad de condiciones crónicas, compartían similares puntos de vista. De su trabajo se deriva que 
no todos los adolescentes con condiciones crónicas tienen las mismas ideas acerca de su enfermedad y del tratamiento. En este sentido los autores identificaron cuatro perfiles en relación con las prioridades y preferencias de los adolescentes sobre el cuidado de la salud, el auto-manejo de la enfermedad y la adherencia al tratamiento. También identificaron aspectos en los que todos los adolescentes coincidían en manifestar su interés, como era el valor que daban a la posibilidad de tener voz y capacidad para tomar decisiones en relación con su cuidado.

La incorporación de la perspectiva de los adolescentes en el diseño de los entornos y sistemas de cuidado de su salud en los hospitales representa una mejora en los modelos de cuidado centrados en estos pacientes y favorece el diseño de hospitales más amigables para ellos. Gusella y Ward ${ }^{(7)}$ evaluaron la satisfacción con la hospitalización de un grupo de adolescentes en relación con la privacidad, el contacto y las visitas de sus iguales, la movilidad, la independencia y la continuidad educativa. El análisis de los resultados obtenidos por estos investigadores corroboró la importancia de los aspectos discutidos para los adolescentes hospitalizados. En el mismo sentido se manifiestan los resultados del estudio cualitativo llevado a cabo por Blumberg y Devlin en el que se examinaron las preferencias de chicos y chicas de entre 12 y 14 años, en relación tanto con el diseño físico de los hospitales, como con cuestiones relativas a su privacidad en los mismos, a la ocupación del tiempo de hospitalización o a la política de visitas ${ }^{(8)}$.

A pesar de las investigaciones mencionadas, se reconoce que los adolescentes están subrepresentados en la investigación sobre hospitalización y servicios de salud ${ }^{(8)}$. Este grupo de población es muy poco consultado como consumidor de servicios de salud, incluso aunque sean usuarios importantes de estos servicios y sus preferencias y conceptos de cuidado difieran de los de los adultos ${ }^{(6)}$. La consideración de los adolescentes dentro del sistema hospitalario español presenta, además, una serie de carencias desde el punto de vista de los parámetros internacionales de calidad ${ }^{(9)}$. Conocer y tener en consideración el punto de vista de los adolescentes y sus actitudes hacia los sistemas de organización del cuidado de la salud en los hospitales podría contribuir a corregir estas carencias. 
El objetivo del estudio que se presenta fue conocer las preferencias y las prioridades de los adolescentes participantes en relación con diversos aspectos de su cuidado en el hospital. Las cuestiones sobre las que se querían conocer estas preferencias y prioridades estaban relacionadas con potenciales líneas de mejora que pudieran hacer los hospitales más amigables para estos pacientes y más acordes con las recomendaciones internacionales sobre el cuidado de los menores en las instituciones sanitarias.

\section{Método}

Se llevó cabo un estudio descriptivo mediante cuestionario en el que se utilizó un diseño prospectivo transversal. Se consideraron sujetos elegibles para participar en la investigación todos los pacientes de entre 14 y 17 años que ingresaron en el Hospital Universitario de Salamanca en cualquier unidad, excepto en Psiquiatría y en Ginecología, entre los meses de abril y septiembre de 2009. No se consideró la participación de los pacientes ingresados en las unidades de Ginecología y Psiquiatría porque el tipo de patologías atendidas en estas unidades hace que las problemáticas de estos pacientes sean muy específicas y distintas de las de los adolescentes ingresados en otras unidades. Un técnico del equipo de investigación se puso en contacto con cada uno de los pacientes considerados elegibles, explicándoles el objetivo de la investigación e invitándoles a participar. Si aceptaban, ellos y sus padres o representantes legales debían firmar un consentimiento informado al respecto antes de completar los datos y la información requerida en el cuestionario autoadministrado en formato papel y lápiz que se utilizó. Se les ofrecía la posibilidad de ayudarles si presentaban dificultades de movilidad o de otro tipo. A cada participante se le obsequió con un regalo de material de papelería.

Para la preparación del cuestionario utilizado se tomaron como referencia las líneas de investigación mencionadas sobre preferencias de los adolescentes acerca del cuidado de su salud ${ }^{(2,7,10)}$. Se consideraron además las recomendaciones internacionales sobre el cuidado de los menores en las instituciones sanitarias ${ }^{(9)}$. Ninguna de las investigaciones citadas sobre preferencias de los adolescentes en relación con su cuidado en los hospitales se habían llevado a cabo en España, por lo que se consideró necesario desarrollar un instrumento de recogida de información adaptado a las 
situaciones de los hospitales españoles y, más en concreto, a las características del cuidado de los pacientes adolescentes en ellos ${ }^{(11)}$. El cuestionario incluía datos respecto a la edad, sexo y experiencia de hospitalización, 15 preguntas (escala Likert) para valorar de 1 a 5 la importancia concedida a diferentes aspectos de la estancia hospitalaria, 6 preguntas con varias opciones de respuesta sobre las preferencias por tipo y compañero de habitación y por acompañante nocturno en el hospital, y 5 preguntas abiertas para indicar aspectos positivos y negativos de la hospitalización y sugerencias para mejorarla. Se realizó un pilotaje cognitivo del cuestionario con un grupo de adolescentes para asegurar la correcta comprensión de todos su ítems. La versión completa del cuestionario se pueden consultar en http://gredos2.usal.es/jspui/handle/10366/70662.

\section{Estrategia de análisis}

Se efectuó un análisis descriptivo de las respuestas de los sujetos. Se evaluó la significación estadística de las diferencias observadas entre las respuestas de las chicas y las de los chicos en las preguntas de escala Likert y en las de varias opciones de respuesta. Se utilizó una prueba chi-cuadrado para evaluar la significación estadística de la diferencia entre proporciones y una prueba t para evaluar la significación estadística de las diferencias entre medias. Las pruebas estadísticas se llevaron a cabo con el programa SPSS (versión 15). Se efectuó un análisis de contenido descriptivo y temático de las respuestas de los participantes a las preguntas abiertas. Las categorías y subcategorías utilizadas se establecieron de manera inductiva. La codificación se realizó utilizando el programa de análisis cualitativo Nvivo8.

\section{Resultados}

Aceptaron participar y completaron el cuestionario $96(76 \%)$ de los 126 pacientes adolescentes considerados elegibles, 16 (16\%) estaban ingresados en la unidad de Pediatría del hospital y 80 (84\%) en distintas unidades de adultos. Su distribución por edad y sexo figura en la tabla 1 .

De los 15 ítems cuya importancia para mejorar la hospitalización debían evaluar los participantes (véase tabla 2) los más valorados, por término medio, fueron los 
relativos a que no se retrasaran las pruebas diagnósticas para que la estancia en el hospital fuera lo más breve posible (media 4.55, DT .819, IC95\%: 4.39-4.72), a la amabilidad del personal sanitario (media 4.40, DT .923, IC95\%: 4.21-4.58) y a la presencia permanente de los padres en el hospital (media 4.06, DT 1.159, IC95\%: 3.83 4.30). Excepto en el ítem 9, no hubo diferencias significativas en función del sexo en la valoración de los demás. Este ítem, que hacía referencia a disponer de más sitio para las cosas personales en la habitación, fue valorado de forma más alta por los chicos que por las chicas $\left(\mathrm{t}_{92}=-3.15, \mathrm{p}<.05\right)$. La valoración media de los chicos en él fue de 2.92 (95\% IC:2.58-3.25) y la de las chicas de 2.18 (95\%IC:1.82-2.53).

El 60.4\% (IC95\%: 49.9-70.2) de los adolescentes participantes afirmaron preferir que la habitación del hospital fuese individual (véase tabla 3). En caso de tener que compartir la habitación, el 86.4\% (IC95\%:77.9-92.5) afirmó que preferiría compartirla con alguien de la misma edad, y el 13.5\% (IC95\%:7.4-22.0) afirmó que no le importaba la edad del compañero de habitación. De tener que elegir entre compartir la habitación con alguien de la misma edad o con alguien que tuviera problemas médicos parecidos, el 63.5\% (IC95\%:53.0-73.1) prefería compartir la habitación con alguien de la misma edad, independientemente de sus problemas médicos. El 93.7\% (IC95\%:86.8-97.6) afirmó preferir pasar la noche en el hospital acompañado, y como acompañante nocturno en el hospital el 91.6\% (IC95\%:84.2-96.3) señaló a sus padres. En ninguno de estos aspectos hubo diferencias significativas entre los chicos y las chicas.

El cuestionario incluía tres preguntas abiertas en las que se pedía a los participantes que anotasen sugerencias e iniciativas que podían ayudarles a estar mejor durante su estancia en un hospital. Los adolescentes participantes en esta investigación hicieron un número importante de propuestas y sugerencias para mejorar la hospitalización de los chicos y chicas de su edad. La mayoría de ellas tenían que ver con cuestiones relacionadas con las instalaciones y con los servicios no médicos del hospital, en concreto con los servicios disponibles para la ocupación del tiempo de hospitalización, con el confort de las habitaciones, del mobiliario y de la ropa que tienen que utilizar los pacientes y con la comida del hospital. Prácticamente la mitad de las propuestas estaban relacionadas con la mejora de los servicios de ocio y ocupación del tiempo en el hospital. Los adolescentes sugirieron con insistencia mejorar la tecnología 
de ocio disponible para los pacientes (sobre todo Internet y televisión gratuita en las habitaciones), así como poder participar en actividades de ocio y entretenimiento mientras están hospitalizados. Destaca, asimismo, la sugerencia de contar con espacios de ocio (salas de juegos, para escuchar música, etc.) fuera de sus habitaciones donde pasar parte del tiempo que tienen que estar en el hospital. El sistema de organización de la estancia en el hospital de los pacientes adolescentes también recibe una proporción significativa de sugerencias de mejora. En esta categoría destacan especialmente las sugerencias de mejora que tienen que ver con los compañeros de habitación o de planta de ingreso. Los participantes proponían no ser hospitalizados con personas mayores sino con pacientes de su edad. En la tabla 4 se resume el análisis de contenido descriptivo y temático de todas las sugerencias efectuadas por los participantes. En total se codificaron 194 referencias en 6 categorías, que a su vez se subdividieron en varias subcategorías. La categoría que presenta un mayor número de referencias de codificación es la de "Instalaciones y servicios del hospital" (139 referencias, 71.6\%), y dentro de ella la subcategoría de "servicios de ocio y ocupación del tiempo de hospitalización" (109 referencias, 56.2\%).

Las respuestas de los sujetos a las preguntas abiertas sobre aspectos positivos y negativos de la hospitalización también se transcribieron y se codificaron temáticamente. En las respuestas a los aspectos positivos de la estancia en el hospital se codificaron 86 referencias en 7 categorías (véase tabla 5). El trato del personal sanitario aparece como el aspecto positivo de la hospitalización mencionado con más frecuencia (43 referencias, 50.0\%). En las respuestas que dieron los sujetos participantes a la pregunta abierta sobre los aspectos negativos de la hospitalización se codificaron 94 referencias en 9 categorías (véase tabla 6). "Instalaciones y servicios del hospital" fue la categoría en la que se codificaron mayor número de referencias (35 referencias, 37.2\%), que hacían mención a la comida del hospital (15 referencias, 16.0\%), a la falta de confort de habitaciones, mobiliario y ropa (10 referencias, 10.6\%) y las carencias en los servicios de ocio para los pacientes adolescentes (10 referencias, 10.6\%). 


\section{Discusión}

El propósito de este estudio fue conocer las preferencias y prioridades de una muestra de adolescentes hospitalizados en relación con diversos aspectos que pudieran mejorar su estancia en el hospital. De los resultados obtenidos merecen destacarse tres aspectos. En primer lugar la importancia que los participantes atribuyeron a la amabilidad y al trato del personal sanitario para mejorar su experiencia de hospitalización. Las cuestiones que tienen que ver con los componentes interpersonales del cuidado ya habían aparecido en varios estudios como aspectos muy valorados por los adolescentes cuando hacen un juicio de la calidad de los cuidados de salud que reciben ${ }^{(10,12)}$, sin que en ello se observaran diferencias señaladas con respecto a los pacientes adultos.

En segundo lugar, destaca la marcada preferencia de los participantes por compartir habitación con alguien de edad parecida. Compartir la hospitalización con pacientes de similar edad es un aspecto significativo de la atención de niños y adolescentes en los hospitales, y como tal recogido en las recomendaciones internacionales sobre el cuidado de los menores en las instituciones sanitarias ${ }^{(9)}$. Desde una perspectiva evolutiva, la adolescencia se entiende como el período de transición desde la infancia a la vida adulta e implica importantes cambios biológicos, psicológicos y sociales que deben tenerse en cuenta en la prestación de servicios sanitarios a este grupo de población ${ }^{(13)}$. Existe evidencia de que los jóvenes que son atendidos en unidades específicas para adolescentes manifiestan una mayor satisfacción con el cuidado recibido que los que ingresan en unidades pediátricas o de adultos ${ }^{(14)}$. A pesar de esta evidencia y de las recomendaciones en apoyo al tipo de cuidados que se prestan en estas unidades a los jóvenes ${ }^{(15,16)}$, en España no existen apenas unidades de ingreso hospitalario no psiquiátrico para adolescentes, y en la mayoría de los hospitales españoles los pacientes adolescentes que sobrepasan la edad pediátrica ingresan en unidades de adultos, en las que comparten planta, y a menudo habitación, con personas con patologías similares pero con grandes diferencias de edad respecto a ellos ${ }^{(11)}$. Se observa, pues, un notable desajuste entre las preferencias de estos pacientes, que como se ha señalado coinciden con las recomendaciones al respecto efectuadas a nivel internacional, y los sistemas efectivos de cuidado de los mismos en los hospitales españoles. 
Finalmente, merece también resaltarse la importancia concedida a las oportunidades de entretenimiento en el hospital por parte de los adolescentes hospitalizados participantes, que sugirieron con insistencia mejorar la tecnología de ocio disponible para los pacientes (sobre todo Internet y televisión gratuita en las habitaciones), así como poder participar en actividades de ocio y entretenimiento mientras están hospitalizados. Consideramos este aspecto particularmente significativo, por cuanto la ocupación del tiempo en el hospital con actividades adecuadas a las edad del paciente ha manifestado un importante desarrollado en el ámbito de la hospitalización pediátrica, pero está escasamente abordado en el ámbito de la hospitalización adulta (17). Ofrecer al paciente la oportunidad de participar en actividades adaptadas a su edad y condiciones de salud durante su estancia en el hospital implica responder a sus necesidades de cuidados psicológicos y sociales, hasta el punto de considerar la forma de pasar el tiempo en el hospital una de las dimensiones de mejora de la salud para los pacientes menores de edad $(8,18,19)$.

Creemos que nuestro estudio tiene varias limitaciones. En primer lugar, no se han considerado las diversas condiciones clínicas de los participantes. Resultaría importante evaluar preferencias y prioridades de los adolescentes teniendo en cuenta sus patologías, el carácter crónico o agudo de las mismas, su gravedad y pronóstico, y el grado en el que estas patologías pueden afectar a la vida cotidiana de los adolescentes. Consideramos también otra limitación que los adolescentes que participaron en este estudio recibían atención sanitaria en un único hospital. Las características de este hospital representan un promedio de los hospitales españoles de su tamaño y en él se sigue la norma general de hospitalizar a los pacientes en unidades de adultos a partir de 14-15 años, como en el $80 \%$ de los hospitales nacionales, según los datos disponibles ${ }^{(11,}$ 20). Aún así, resultaría conveniente realizar estudios multicentro para confirmar los resultados en este aspecto. A pesar de estas limitaciones, consideramos que los resultados obtenidos nos permiten concluir que los adolescentes hospitalizados participantes manifestaron una perspectiva de usuarios del hospital potencialmente útil en el diseño de estrategias de mejora de la hospitalización de estos pacientes. 


\section{Referencias bibliográficas}

1. Schattner A, Bronstein A, Jellin N. Information and shared decision-making are top patients' priorities. BMC Health Serv Res. 2006;6(1):21.

2. Britto MT, Slap GB, DeVellis RF, Hornung RW, Atherton MS, Knopf JM, et al. Specialists understanding of health care preferences of chronically ill adolescents. J Adolesc Health. 2007;40:334-41.

3. Grol R, Wensing M, Mainz J, Jung H, Ferreira P, Hearnshaw H, et al. Patients in Europe evaluate general practice care: an international comparison. Br J Gen Pract. 2000;50:882-7.

4. Wensing M, Elwyn G. Research on patients`views in the evaluation and improvement of quality of care. Qual Saf Health Care. 2002;11(2):153-7.

5. Wray J, Maynard L. Specialist cardiac services: what do young people want? Cardiol Young. 2008;18(6):569-74.

6. Jedeloo S, Staa Av, Latour JM, Exel JAv. Preferences for health care and selfmanagement among Dutch adolescent with chronic conditions: a Q-methodological investigation. Int J Nurs Stud. 2010;47(5):593-603.

7. Gusella JL, Ward AM. The experience of hospitalized adolescent: how well do we meet their developmental needs? Child Health Care. 1998;27(2):131-45.

8. Blumberg R, Devlin AS. Design issues in hospitals: The adolescent client. Environ Behav. 2006;38(3):293-317.

9. Ullán AM, Belver MH. Cuando los pacientes son niños: Humanización y calidad en la hospitalización pediátrica. Madrid: Eneida; 2008.

10. Britto MT, DeVellis RF, Hournung RW, DeFriee GH, Atherton HD, Slap GB. Health care preferences and priorities of adolescentes with chronic illnesses. Pediatrics. 2004;114(5):1272-80.

11. Ullán AM, Gonzalez R, Manzanera P. El cudiado de los adolescentes en los hospitales españoles: los pacientes invisibles. Rev Calid Asist. 2010;25(3):146-52.

12. Hoffman ND, Freeman K, Swann S. Healthcare preferences of lesbian, gay, bisexual, transgender and questioning youth. J Adolec Health. 2009;45:222-9.

13. Hidalgo MI, González MP, Montón JL. Atención a la adolescencia. FMC Form Med Contin Aten Prim. 2006;13:1-49. 
14. Viner RM. Do Adolescent Inpatient Wards Make a Difference? Findings From a National Young Patient Survey. Pediatrics. 2007;120(4):749-55.

15. Bryon M, Madge S. Transition from paediatric to adult care: psychological principles. J R Soc Med. 2001;94(suppl. 40):5-7.

16. Southall DP, Burr S, Smith RD, Bull DN, Radford A, Williams A, et al. The Child-Friendly Healthcare Initiative (CFHI): Healthcare Provision in Accordance With the UN Convention on the Rights of the Child. Pediatrics. 2000;106:1054 - 64.

17. Ullán AM, Belver MH. Jugar para estar mejor: el juego de los niños en los hospitales. En: Belver MH, Ullán AM, editors. La creatividad a través del juego.

Salamanca: Amarú Ediciones; 2006. p. 249-72.

18. Avila N. Talleres de arte con adolescentes hospitalizados. Una experiencia de comunicación. Revista venezolana de educación. 2009;45:449-56.

19. Pascale P, Ávila N. Una experiencia de creatividad con adolescentes hospitalizados. Arte Individ Soc. 2007;19:207-46.

20. Fernández E, Gándara S, Morato M. Hospital Universitario de Salamanca: investigación y mejora de la calidad. En: Ullán AM, Belver MH, editors. Cuando los pacientes son niños: Humanización y calidad en la hospitalización pediátrica. Madrid: Eneida; 2008. p. 112-6. 
TABLA 1. Distribución de la muestra por edad y sexo

\begin{tabular}{ll}
\cline { 2 - 2 } & $\mathrm{n}(\%)$ \\
\hline Edad & \\
14 años & $25(26,0 \%)$ \\
15 años & $18(18,8 \%)$ \\
16 años & $25(26,0 \%)$ \\
17 años & $28(29,2 \%)$ \\
Sexo & \\
Chicas & $45(46,9 \%)$ \\
Chicos & $51(53,1 \%)$ \\
\hline
\end{tabular}


TABLA 2. Medias, desviaciones típicas (DT) e intervalos de confianza de las valoraciones de los ítems de la primera columna en cuanto a su importancia para mejorar la hospitalización de los adolescentes en una escala de 1, poco importante, a 5, muy importante.

\begin{tabular}{|c|c|c|c|c|}
\hline & \multicolumn{4}{|c|}{$\begin{array}{l}\text { 95\% Intervalo de } \\
\text { confianza }\end{array}$} \\
\hline & Media & DT & Inferior & Superior \\
\hline $\begin{array}{l}\text { Ítem } 15 \text {. Que no se retrasen las pruebas diagnósticas } \\
\text { para que la estancia en el hospital sea lo más breve } \\
\text { posible }\end{array}$ & 4.55 & 0.819 & 4.39 & 4.72 \\
\hline Ítem 4. La amabilidad del personal sanitario & 4.40 & 0.923 & 4.21 & 4.58 \\
\hline Ítem 2. La presencia permanente de los padres & 4.06 & 1.159 & 3.83 & 4.30 \\
\hline Ítem 10. Tener TV gratuita en la habitación & 4.03 & 1.162 & 3.79 & 4.27 \\
\hline Ítem 8. Acceso a Internet en el hospital & 4.01 & 1.284 & 3.75 & 4.27 \\
\hline $\begin{array}{l}\text { Ítem } 5 \text {. Información médica clara que el paciente } \\
\text { pueda comprender }\end{array}$ & 4.00 & 1.107 & 3.77 & 4.23 \\
\hline Ítem 1. Las visitas de los amigos & 3.82 & 1.130 & 3.59 & 4.05 \\
\hline $\begin{array}{l}\text { Ítem 6. Espacios fuera de la habitación para estar } \\
\text { con los amigos o con otros pacientes de la misma } \\
\text { edad }\end{array}$ & 3.73 & 1.039 & 3.52 & 3.95 \\
\hline Ítem 7. Salas para escuchar música, ver TV, leer, etc. & 3.61 & 1.294 & 3.35 & 3.88 \\
\hline $\begin{array}{l}\text { Ítem 11. Que se llame siempre a la puerta antes de } \\
\text { entrar } \\
\text { Ítem 3. Estar ingresado con chicos de la misma edad }\end{array}$ & 3.60 & 1.162 & 3.36 & 3.84 \\
\hline & 3.39 & 1.100 & 3.17 & 3.62 \\
\hline $\begin{array}{l}\text { Item } 13 \text {. No tener que compartir la habitación con } \\
\text { pacientes adultos }\end{array}$ & 3.35 & 1.442 & 3.05 & 3.64 \\
\hline $\begin{array}{l}\text { Ítem 12. Que hubiera en el hospital profesores para } \\
\text { ayudar a que los pacientes no se retrasaran en los } \\
\text { estudios } \\
\text { Ítem 14. Que la habitación tuviera una decoración } \\
\text { adaptada a los gustos de los chicos de su edad }\end{array}$ & 3.04 & 1.398 & 2.76 & 3.33 \\
\hline & 2.80 & 1.292 & 2.53 & 3.06 \\
\hline personales en la habita & 2.63 & 1.209 & 2.38 & 2.88 \\
\hline
\end{tabular}

Nota. Los ítems están ordenados según el criterio de media descendente. El número de cada ítem se corresponde con el número de orden de presentación del mismo en el cuestionario 
TABLA 3. Número y porcentaje de sujetos que señalaron cada una de las opciones de respuesta en las preguntas relativas a la preferencia por tipo, compañero de habitación y acompañante en el hospital.

Enunciado de la pregunta y opciones de respuesta

n $(\%)$

¿Cómo preferirías que fuese tu habitación en el hospital?

Individual

$58(60.4 \%)$

Compartida

$18(18.8 \%)$

Me da lo mismo

$20(20.8 \%)$

Si tienes que compartir la habitación del hospital con otro paciente ¿prefieres que sea alguien de tu edad o te da lo mismo la edad de tu compañero de habitación?

Con alguien de la misma edad

Me da lo mismo la edad del compañero

$13(13.5 \%)$

Si tienes que compartir la habitación del hospital con otro pacientes ¿prefieres que sea con alguien con problemas médicos parecidos a los tuyos o ese aspecto te da lo mismo si no supone un riesgo para ti?

Con alguien con problemas médicos parecidos a los míos

Ese aspecto me da lo mismo

Si tienes que compartir la habitación del hospital con otro paciente, qué preferirías ¿que tuviese problemas médicos similares a los tuyos, aunque su edad fuese distinta de la tuya, o que tuviese más o menos tu edad, al margen de los problemas médicos que pudiera tener, siempre que no supusiera un riesgo para ti?

Compañero con problemas médicos parecidos

Compañero de edad parecida

$61(63.5 \%)$

Si tienes que pasar la noche en el hospital ¿cómo prefieres pasarla?

Acompañado

$90(93.7 \%)$

Solo

$5(5.2 \%)$

¿Quién prefieres que te acompañe durante la noche en el hospital? ${ }^{a}$

Padres

$88(91.6 \%)$

Otros familiares

$14(14.6 \%)$

Amigos

$7(7.3 \%)$

Personal sanitario profesional

$1(1.0 \%)$

${ }^{a}$ Los porcentajes suman más de 100 porque la pregunta admitía varias respuestas 
TABLA 4. Referencias de codificación en cada una de las categorías en que se catalogaron las respuestas a las preguntas abiertas relativas a qué creían los participantes que podía ayudar a los adolescentes a estar mejor durante su estancia el hospital

\begin{tabular}{cll} 
& \multicolumn{2}{l}{$\begin{array}{l}\text { Referencias de } \\
\text { codificación }\end{array}$} \\
Categorías de codificación y subcategorías & $\mathrm{N}=194$ & \\
& $\mathrm{n}$ & $(\%)$ \\
\hline Instalaciones y servicios del hospital & 139 & $(71.6 \%)$ \\
Servicios de ocio y ocupación del tiempo & 109 & $(56.2 \%)$ \\
Tecnología de ocio & 68 & $(35.1 \%)$ \\
Internet & 25 & $(12.9 \%)$ \\
TV & 16 & $(8.3 \%)$ \\
Ordenadores & 13 & $(6.7 \%)$ \\
Música & 7 & $(3.6 \%)$ \\
Videojuegos & 7 & $(3.6 \%)$ \\
Actividades de ocio y entretenimiento & 17 & $(8.8 \%)$ \\
Espacios de ocio en el hospital & 17 & $(8.8 \%)$ \\
Lectura y películas & 7 & $(3.6 \%)$ \\
Confort de habitaciones, mobiliario y ropa & 21 & $(10.8 \%)$ \\
Comida & 9 & $(4.6 \%)$ \\
Organización de la estancia en el hospital & 35 & $(18.0 \%)$ \\
Compañeros de habitación y o de planta & 18 & $(9.3 \%)$ \\
Modo de ocupar el tiempo & 7 & $(3.6 \%)$ \\
Relación con pacientes de la misma edad & 10 & $(5.1 \%)$ \\
Trato del personal sanitario & 3 & $(1.6 \%)$ \\
Apoyo social de familia y amigos & 5 & $(2.6 \%)$ \\
Cuidados sanitarios y recuperación & 2 & $(1.0 \%)$ \\
Atención escolar & 3 & $(1.6 \%)$ \\
Respuestas sin codificar & 7 & $(3.6 \%)$ \\
\hline
\end{tabular}


TABLA 5. Referencias de codificación en cada una de las categorías en que se catalogaron las respuestas a la pregunta abierta sobre aspectos positivos de la hospitalización

\begin{tabular}{lll} 
& \multicolumn{2}{l}{$\begin{array}{l}\text { Referencias de } \\
\text { codificación }\end{array}$} \\
Categorías de codificación & $\mathrm{N}=86$ & \\
& $\mathrm{n}$ & $(\%)$ \\
\hline Trato del personal sanitario & 43 & $(50.0 \%)$ \\
Atención médica y recuperación & 13 & $(15.1 \%)$ \\
Instalaciones y servicios del hospital & 9 & $(10.5 \%)$ \\
Apoyo social de familia y amigos & 6 & $(7.0 \%)$ \\
Nada positivo & 5 & $(5.8 \%)$ \\
Conocer relacionarte con otra gente & 2 & $(2.3 \%)$ \\
Actividades de entretenimiento para ocupar el tiempo & 3 & $(3.5 \%)$ \\
Respuestas sin codificar & 5 & $(5.8 \%)$ \\
\hline
\end{tabular}


TABLA 6. Referencias de codificación en cada una de las categorías y subcategorías en que se catalogaron las respuestas sobre los aspectos negativos de la hospitalización

\begin{tabular}{lll} 
& \multicolumn{2}{l}{ Referencias de } \\
codificación \\
Categorías de codificación & $\mathrm{N}=94$ & \\
& $\mathrm{n}$ & $\%$ \\
\hline Instalaciones y servicios del hospital & 35 & $(37.2 \%)$ \\
$\quad$ Servicios de ocio & 10 & $(10.6 \%)$ \\
$\quad$ Incomodidad de habitaciones, mobiliario o ropa & 10 & $(10.6 \%)$ \\
$\quad$ Comida & 15 & $(16.0 \%)$ \\
Organización de la estancia en el hospital & 14 & $(14.9 \%)$ \\
$\quad$ Compañeros de habitación o planta & 8 & $(8.5 \%)$ \\
$\quad$ Falta de privacidad, de tranquilidad & 3 & $(3.2 \%)$ \\
$\quad$ Horarios del hospital & 3 & $(3.2 \%)$ \\
Aburrimiento & 7 & $(7.5 \%)$ \\
Atención médica, diagnóstico tratamiento & 7 & $(7.5 \%)$ \\
Estar enfermo o sentir dolor & 6 & $(6.4 \%)$ \\
Trato del personal del hospital & 5 & $(5.3 \%)$ \\
Estar en el hospital, no poder salir del hospital o de la & 4 & $(4.3 \%)$ \\
habitación & 4 & $(4.3 \%)$ \\
Todo & 6 & $(6.4 \%)$ \\
Nada & 4 & $(4.2 \%)$ \\
Respuestas in codificar & &
\end{tabular}

